\title{
A RARE CASE OF CENTRAL PLACENTA PREVIA ACCRETA
}

\section{Dr. Pallavi \\ Madhukar \\ Unhale* \\ Dr. Vijay $\mathbf{Y}$. \\ Kalyankar}

\section{Dr. Shrinivas Gadappa}

\section{Dr. Megha}

(Junior Resident)Department of Obstetrics and Gynecology, Government Medical College and Hospital, Aurangabad-431001, Maharashtra, India *Corresponding Author

(Associate Professor)Department of Obstetrics and Gynecology, Government Medical College and Hospital, Aurangabad-431001, Maharashtra, India

(Professor and Head)Department of Obstetrics and Gynecology, Government Medical College and Hospital, Aurangabad-431001, Maharashtra, India (Junior Resident)Department of Obstetrics and Gynecology, Government Medical College and Hospital, Aurangabad-431001, Maharashtra, India

Placenta previa accreta, is a rare occurrence which occurs in $3 \%$ of women diagnosed with placenta previa with massive blood loss in almost every case resulting in cesarean hysterectomy. We report the case of central placenta previa with accreta in a case with no cause known to precipitate it. With aggressive radical surgery, massive transfusion protocol, well prepared to face it unexpectedly and vigilant postoperative management, such cases can be managed successfully.

\section{Introduction:}

Placenta previa is the complete or partial covering of the internal Os of the cervix with placenta. It is a severe complication of pregnancy and is the most common cause of post-partum hemorrhage, which often endangers the lives of pregnant women, can lead to morbidity and mortality of the mother and neonate. A study conducted by B Kavitha, Bansal $\mathrm{H}$ Pota Placenta previa is frequently reported to occur in 1 in 200-250 pregnancies This situation prevents a safe vaginal delivery and requires the delivery of the neonate to be via caesarean delivery.

Placenta accreta is a pathological condition of placentation associated with massive maternal hemorrhage. Initially described in 1937 by Irving and Hertigl as abnormal adherence of placenta to the myometrium due to the partial or complete absence of decidua basalis subsequently redefined by Luke et al as a spectrum of abnormally adherent and invasive placentation disorder. The presence of placenta previa can also increase a woman's risk for placenta accreta spectrum (PAS). A combination of both placenta previa and PAS is therefore further dangerous threatening mother and newborn both.

\section{Case History:}

A 32-year-old woman (gravida 3 para 2 live 2, both delivered normally and no abortions or h/o curettage) with 38.2 weeks of $\mathrm{GA}$ referred in emergency to $\mathrm{GMCH}$, Aurangabad our tertiary care Centre with complaint of first episode of painless fresh vaginal bleeding. No ANC visits in 2 nd and 3 rd trimester with no blood investigations were done. Routine early scan at 17 weeks s/o posterior low lying placenta. Obstetric Ultrasound done in emergency suggested grade 4 placenta previa only. Emergency LSCS for placenta previa in bleeding phase was done. A healthy baby weighing $3.1 \mathrm{~kg}$ was delivered. The placenta was not in incision line over LUS. There was evidence of complete placenta previa and no plane of separation between placenta and uterus with excess bleeding. There was no encroachment on urinary bladder. So, Total Obstetric hysterectomy with placenta in situ was done as a life-saving procedure after taking written valid informed consent. The total intraoperative blood loss was 1.5 liters. Massive transfusion protocol i.e. 4-units of PRBC, 4-units of RDP and 4-units of FFP were given. Post- operative recovery was good and she went home healthy.

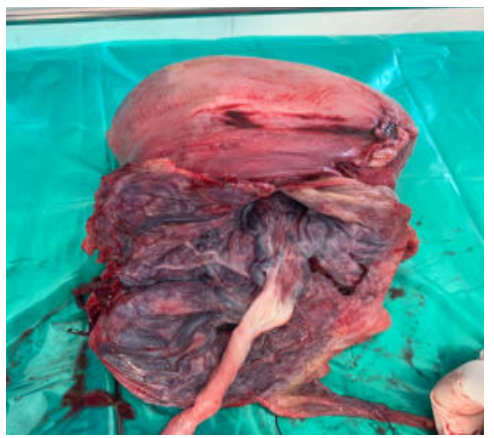

Figure 1-Gross specimen after Obstetric Hysterectomy

\section{Discussion-}

As discussed in American Journal of Obstetrics and Gynecology in June 27 year 2020 by Frances M. AndersonBagga; Angelica Sze, the incidence of placenta previa accreta $^{3,4}$ has increased from approximately $0.8 / 1000$ deliveries in the 1980 's to $3 / 1000$ deliveries in the past decade. In 20 studies studied by Eric Jauniaux, LeneGronbeck and Sally L Collins ${ }^{2}$, out of 6628 cases of placenta previa, 587 were complicated by PAS. Prevalence of previa was $0.56 \%$ whereas the prevalence of placenta previa with PAS was $0.07 \%$. Incidence of PAS in women with placenta previa was $11.10 \%$.

The risk factors for placenta previa are advanced maternal age, multiparity, smoking, cocaine use, prior suction and curettage, assisted reproductive technology, history of cesarean sections and prior placenta previa. ${ }^{5,6,7}$ Usual presentation at the time of admission in $2^{\text {nd }}$ and $3^{\text {rd }}$ trimester is painless vaginal bleeding ${ }^{8}$. In our scenario, patient was a multigravida with painless fresh vaginal bleeding for the first time with no risk factor to explain placenta previa with PAS. 
Nearly $90 \%$ of placentas identified as "low lying " will ultimately resolve by the third trimester. Follow up sonogram is recommended at 28-32 weeks of gestation to look for persistent placenta previa. Ultrasonography has very high sensitivity of about $93 \%$ and specificity of about $71 \%$ in the diagnosis of placenta accreta spectrum (PAS) ${ }^{9}$. The role of MRI has increased in recent years in its diagnosis. In our case, ultrasound USG at 17 weeks showed low lying placenta and 38.2 weeks suggested placenta previa. PAS was not reported.

Bedrest, reduced activity and avoidance of intercourse are commonly recommended in early diagnosed placenta previa cases and if the vaginal bleeding subsides for more than 48 hours and fetus is judged to be healthy then inpatient monitoring is continued or patient can be discharged for outpatient management. Also, outpatient vs. inpatient management depends on the stability of the patient, number of episodes of bleeding, proximity to the hospital, as well as compliance. Frances M. Anderson-Bagga suggested that with the early diagnosis of dangerous placenta previa, the patient can be scheduled for elective delivery at 36-37 weeks via Csection but patients with excessive or continuous vaginal bleeding should be delivered via C-Section in emergency situation regardless of gestational age. This case presented for the first time with first episode of bleeding PV at 38.2 weeks and was taken for emergency caesarean section.

A study conducted by Frances M. Anderson-Bagga; Angelica Sze suggested that conservative management is also one of the options in managing patients with PAS if the patient desires fertility. The placenta can be left in situ until there is devascularization of the placental bed so that the remaining placental tissue may either be safely removed or resorbs itself. However there is high recurrence rate of placenta accreta in the next pregnancy ranging from 17 to $29 \%{ }^{10}$. A review by Kenji Tanimura and Hideto Yamada submitted on march $29^{\text {th }} 2018$ which summarized the conservative management of 60 women with placenta accreta, showed that infection occurred in 11 women (18\%), bleeding in 21 (35\%), and disseminated intravascular coagulation in 4(7\%). Therefore conservative approach should be considered only when women are willing to accept the risks involved. In our case patient was multigravida with previous two live issues so option of obstetric hysterectomy was chosen.

Preterm birth, vaginal bleeding, placenta accreta, placental abruption, bladder injuries during hysterectomies, ICU admissions, septicemia and even death are the common complications of placenta previa accreta Despite the early and accurate prenatal diagnosis, hysterectomy remains the most common surgical procedure in cases of placenta previa accreta. In our case emergency $\mathbf{C}$ - section was immediately proceeded to obstetric hysterectomy. So, during surgery we transfused massive transfusion protocol and retrograde filled up the urinary bladder with normal saline to delineate the bladder and operated on the uterus to avoid any injury to it.

Morbidity and mortality after peripartum hysterectomy are significant. A study conducted by Lovina S.M.Machado has nationwide sample of women in USA who underwent obstetric hysterectomy, reported a mortality rate of 0 to $12.5 \%$, whereas morbidity ranged from $26.5 \%$ to $31.5 \%$. Massive blood loss, injuries to genitourinary tract are most common, with reported rates of cystotomy of $6-29 \%$ and ureteric injuries in up to $7 \%$ of women ${ }^{12}$. Postoperatively febrile complications, bowel dysfunctions are relatively frequent. Among women who require re-exploration, approximately three quarters cases are to control bleeding, whereas the remainder are procedures for the repair of operative injuries. In our case patient had no intraoperative complications and remained healthy postoperatively.

|www.worldwidejournals.com

\section{Conclusion}

Placenta previa accreta though commonly seen in cases having predisposing high risk factors, it may also be seen unusually without any precipitating cause. So, one should be adequately prepared to surgically face it, unexpectedly with team approach , Massive transfusion protocol, timely surgery and avoiding injury to vital structures can reduce the morbidity and mortality.

\section{References:}

1. Irving C, Hertig AT. A study of placenta accreta. surgery. GynecolObstet 1937;64:178-200

2. Luke RK, Sharpe JW, Greene RR. Placenta accreta: the adherent or invasive placenta. Am J ObstetGynecol1966;95:660-8. 10.1016/S00029378(16)34741-X

3. K. M. Flood, S. Said, M. Geary, M. Robson, C. Fitzpatrick, and F. D. Malone, "Changing trends in peripartum hysterectomy over the last 4 decades," American Journal of Obstetrics and Gynecology, vol. 200, no. 6, pp. 632.el-632.e6,2009.

4. S. Wu, M. Kocherginsky, and J. U. Hibbard, "Abnormal placentation: twentyyear analysis," American Journal of Obstetrics and Gynecology, vol. 192, no. 5, pp. 1458-1461,2005

5. Martinelli KG, Garcia ÉM, Santos Neto ETD, Gama SGND. Advanced maternal age and its association with placenta praevia and placental abruption: a meta-analysis. Cad Saude Publica.2018 Feb 19;34(2):e00206116.

6. Silver RM. Abnormal Placentation: Placenta Previa, Vasa Previa, and Placenta Accreta. Obstet Gynecol.2015 Sep;126(3):654-68

7. Jing L,Wei G, Mengfan S, Yanyan H. Effect of site of placentation on pregnancy outcomes in patients with placenta previa. PLoS ONE. 2018;13(7):e0200252

8. Pedigo R. First trimester pregnancy emergencies: recognition and management. Emerg Med Pract. 2019 Jan;2 1(1):1-20

9. Silver RM, Branch DW. Placenta Accreta Spectrum. N. Engl. J. Med. 2018 Apr 19;378(16):1529-1536

10. MacGibbon A, Ius YM. Conservative Management of Abnormally Invasive Placenta Previa after Midtrimester Foetal Demise. Case Rep Obstet Gynecol. 2018;2018:7478437

11. Timmermans S, van Hof AC,Duvekot JJ. Conservative management of abnormally invasive placentation. Obstetrical and Gynecological Survey. 2007;62:529-539

12. Eller AG, Porter TF,SoissonP,SilverRM.Optimal management strategies for placenta accreta. BJOG2009;116:648-54. 